\title{
Proposta de Adaptação de uma Técnica de Sincronização de Símbolo para Aplicação em uma Metodologia de Medição de Canais PLC
}

\author{
Fernando Augusto Flores Villaça, Ândrei Camponogara, Thiago Rodrigues Oliveira e Moisés Vidal Ribeiro .
}

\begin{abstract}
Resumo-Neste trabalho, propõe-se uma adaptação da técnica de sincronismo temporal baseada na modulação por divisão ortogonal em frequência (orthogonal frequency division multiplexing - OFDM) proposta por Schmidl \& Cox para uso em metodologia de medição de canais de comunicação via rede de energia elétrica (do inglês, power line communication - PLC). Resultados numéricos comparativos mostram que a técnica adaptada alcança os melhores resultados e que, diferente da técnica originalmente proposta, ela não depende de ajustes empíricos de parâmetros.
\end{abstract}

Palavras-Chave-Comunicação via rede de energia elétrica, sincronização, estimação de canal.

Abstract-This paper proposes a modification of the timing synchronization technique based on orthogonal frequency division multiplexing (OFDM) scheme proposed by Schmidl \& Cox to be applied within a methodology for estimating power line communication (PLC) channels. Comparative numerical results show that the modified technique shows the best performance and, differently of the technique originally proposed, it is not dependent of empirical parameters tuning.

Keywords - Power line communication, synchronization, channel estimation.

\section{INTRODUÇÃO}

O uso de redes de energia elétrica para a transmissão de dados, conhecido como comunicação via rede de energia elétrica (do inglês, power line communication) (PLC), vem sendo investigado há algum tempo. Assim como em outros meios de comunicação, as medições desses canais são de extrema importância para o desenvolvimento de tecnologias PLC cada vez melhores. Uma abordagem bastante aplicada para a realização das medições em canais PLC é aquela baseada na utilização do analisador de rede vetorial (do inglês, vector network analizer) (VNA) [1], [2]. No entanto, ela oferece uma série de limitações, como por exemplo, um intervalo de tempo muito grande entre duas medições do canal PLC, dificultando, assim, a estimação do tempo de coerência desse canal. Para superar essas limitações, pode-se utilizar

O presente trabalho foi realizado com apoio da Coordenação de Aperfeiçoamento de Pessoal de Nível Superior (CAPES) - Código de financiamento 001, do Ministério da Educação (MEC), do Conselho Nacional de Desenvolvimento Científico e Tecnológico $(\mathrm{CNPq})$, do Instituto Nacional de Energia Elétrica (INERGE) e da Fundação de Amparo à Pesquisa do Estado de Minas Gerais (FAPEMIG).

Fernando Augusto Flores Villaça, Ândrei Camponogara, e Moisés Vidal Ribeiro; Universidade Federal de Juiz de Fora (UFJF); endereços de e-mail: \{fernando.flores, andrei.camponogara, mribeiro\}@engenharia.ufjf.br.

Thiago Rodrigues Oliveira; Instituto Federal de Educação, Ciência e Tecnologia do Sudeste de Minas Gerais, endereço de e-mail: thiago.oliveira@ifsudestemg.edu.br equipamentos distintos para a geração e aquisição dos sinais aplicados no processo de estimação do canal PLC através do emprego de uma abordagem baseada em sounding [3], [4]. Nesse sentido, em [4] foi proposta uma metodologia de medição de canais PLC baseada em sounding que considera o uso de um sinal de referência composto por sucessivos símbolos modulação por divisão ortogonal de frequência hermitiana simétrica (do inglês, Hermetian symmetric orthogonal frequency division multiplexing) (HS-OFDM). Tal abordagem obtém uma estimativa de canal PLC válida para cada símbolo HS-OFDM recebido, que corresponde a um intervalo de tempo muito menor se comparado com aquele demandado pela medição baseada em VNA.

A metodologia da medição proposta em [4] é composta basicamente de quatro etapas: sincronização de símbolo, estimação do erro de frequência de amostragem, estimação da resposta em frequência do canal (do inglês, channel frequency response) (CFR) e enriquecimento da CFR. A sincronização de símbolo permite estimar o início do sinal de referência no receptor, o qual é utilizado para estimar a resposta ao impulso do canal (do inglês, channel impulse response) (CIR) ou a CFR do canal. Dessa forma, ela é uma etapa bastante importante, uma vez que se não for realizada de forma apropriada pode resultar em severa degradação das estimativas de CFR [5].

$\mathrm{Na}$ presente contribuição, considera-se apenas a etapa de sincronização de símbolo e, portanto, propõe-se uma versão adaptada da técnica apresentada por Schmidl \& Cox [6] para ser aplicada na metodologia de medição de canais PLC, comparando-a com a técnica de sincronização de símbolo utilizada em [4]. É importante ressaltar que ambas as técnicas consideram a aplicação de sinais de referência do tipo OFDM para a estimação do canal. Elas são avaliadas através de simulações numéricas realizadas com estimativas de canais PLC reais e considerando diferentes cenários em termos de relação sinal-ruído (do inglês, signal to noise ratio) (SNR). Resultados numéricos mostram que a adaptação proposta na técnica de Schmidl \& Cox [6] resulta em melhores resultados do que aquela apresentada em [4].

O restante do trabalho é organizado da seguinte forma. A Seção II apresenta o modelo do sistema de transmissão utilizado para a avaliação das técnicas de sincronização de símbolo. A Seção III desenvolve a formulação matemática tanto para técnica presente na literatura quanto para a técnica adaptada. Em seguida, a Seção IV apresenta e analisa os resultados numéricos que comprovam as vantagens da proposta introduzida. Por fim, a Seção V apresenta as considerações 


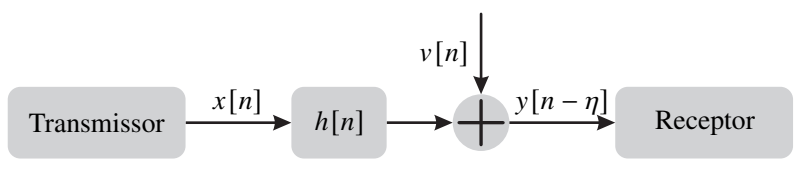

Fig. 1. Diagrama de blocos do sistema PLC considerando o equivalente discreto do modelo do canal na banda base.

finais.

\section{Modelo do Sistema}

Para se obter estimativas de canais PLC através da metodologia de medição discutida em [4], considera-se o arranjo de medição representado de forma simplificada pelo diagrama de blocos mostrado na Fig. 1. De acordo com o diagrama de blocos, o sinal recebido no receptor pode ser representado por

$$
y\left[n-\eta_{d}\right]=\sum_{m=-\infty}^{\infty} x[m] h\left[n-\eta_{d}-m\right]+v[n],
$$

de forma que $\{x[n]\}$ representa a sequência de referência, $\{h[n]\}$ é a resposta ao impulso do canal PLC, $\{v[n]\}$ é o ruído aditivo, e $\eta_{d} \in \mathbb{Z}$ é uma variável aleatória que representa o atraso entre o sinal recebido e a referência temporal local no receptor. Observe que ambos $\{x[n]\}$ e $\{v[n]\}$ são considerados processos aleatórios estacionários no sentido amplo e independentes. Além disso, assumindo-se que as estimativas do canal PLC são obtidas em um intervalo de tempo inferior ao seu tempo de coerência, considera-se que $\{h[n]\}_{n=0}^{L_{h}-1}$ é linear e invariante no tempo.

Como mencionado anteriormente, a sequência de referência $\{x[n]\}$ é formada por uma sequência de sucessivos símbolos HS-OFDM. Esses símbolos são gerados tomando-se a inversa da transformada discreta de Fourier (do inglês, inverse discrete Fourier transform) (IDFT) dos $i$-ésimos sub-símbolos $\mathbf{X}_{i} \in$ $\mathbb{C}^{N \times 1}$, em que $N \in \mathbb{Z}_{+}$é o número de subportadoras. Antes de ser processado pela IDFT, $\mathbf{X}_{i}$ é mapeado no vetor $\mathbf{X}_{\text {map }, i}=$ $\left[X_{\text {map }, i}[0], X_{\text {map }, i}[1], \ldots, X_{\text {map }, i}[2 N-1]\right]^{T}$, de forma que

$$
X_{\text {map }}[k]= \begin{cases}X_{i}[k-1], & k=1, \ldots, N-1 \\ \mathfrak{R}\left\{X_{i}[N-1]\right\}, & k=0 \\ \mathfrak{J}\left\{X_{i}[N-1]\right\}, & k=N \\ X_{i}^{*}[2 N-k-1], & k=N+1, \ldots, 2 N-1,\end{cases}
$$

em que $\mathfrak{R}\{\cdot\}$ e $\mathfrak{I}\{\cdot\}$ representam as partes reais e imaginárias de um dado número complexo, respectivamente, e $\{\cdot\}^{*}$ denota o operador conjugado complexo. O mapeamento de $\mathbf{X}_{i}$ em $\mathbf{X}_{\text {map }, i}$ resulta em $\mathbf{x}_{i} \in \mathbb{R}^{2 N \times 1}$, de maneira que $\mathbf{x}_{i}=$ $\frac{1}{\sqrt{2 N}} \mathbf{W}^{\dagger} \mathbf{X}_{\text {map }, i}$, com $\mathbf{W} \in \mathbb{C}^{2 N \times 2 N}$ representando a matriz da transformada discreta de Fourier (do inglês, discrete Fourier transform) (DFT) e $\{\cdot\}^{\dagger}$ o operador hermitiano simétrico. Com o objetivo de combater a degradação dos símbolos HS-OFDM devido à interferência inter-simbólica gerada pelo canal PLC, é anexado ao início de cada símbolo um prefíxo cíclico (do inglês, cyclic prefix) (CP) de comprimento $L_{c p}$, em que $L_{c p} \geq L_{h}-1$, de forma que a representação vetorial do $i$-ésimo símbolo HS-OFDM resulta em $\mathbf{x}_{c p, i}=\left[x_{i}[2 N-\right.$
$\left.\left.L_{c p}\right], x_{i}\left[2 N-L_{c p}+1\right], \ldots, x_{i}[0], x_{i}[1], \ldots, x_{i}[2 N-1]\right]^{T}$ $\mathrm{e}$, consequentemente, sua versão no tempo discreto é dada por

$$
x[n]=\sum_{i=-\infty}^{\infty} \sum_{j=0}^{2 N+L_{c p}-1} x_{c p, i}[j] \delta\left[n-i\left(2 N+L_{c p}\right)-j\right],
$$

em que $x_{c p, i}[j]$ é o $j$-ésimo elemento de $\mathbf{x}_{c p, i} \in \mathbb{R}^{\left(2 N+L_{c p}\right) \times 1}$ e $\delta[\cdot]$ representa o delta de Kronecker.

A etapa de sincronização de símbolo pode ser expressa por

$$
y[n]=S\left(y\left[n-\eta_{d}\right]\right),
$$

de maneira que $S(\cdot)$ é um operador responsável por obter $\{y[n]\}$ de forma a corrigir $\eta_{d}$. A obtenção de $\{y[n]\}$ se dá por meio do uso de técnicas de sincronização de símbolo, que serão exploradas na seção III.

\section{SINCRONIZAÇÃO DE SÍMBOLO}

Nesta seção são apresentadas duas técnicas de sincronização de símbolo utilizadas para a estimação da amostra inicial da sequência de referência obtida pelo receptor, $n_{\text {sinc }} \in \mathbb{Z}$. A primeira trata-se da técnica discutida em [4], a qual faz uso da correlação entre o $\mathrm{CP}$ e a sua réplica em um símbolo HS-OFDM para estimar $n_{\text {sinc }}$. Já a segunda diz respeito a uma nova proposta introduzida neste trabalho, em que se propõe a adaptação da técnica discutida em [6] para então aplica-lá na metodologia de medição de canais PLC. Observe que essa técnica faz uso da correlação entre duas metades iguais de um símbolo OFDM para estimar $n_{\text {sinc }}$. É importante mencionar que, no contexto de metodologia de medição, não se faz necessária a obtenção precisa de $n_{\operatorname{sinc}}$, pois como se prioriza a estimação da magnitude da CFR, $n_{\text {sinc }}$ apenas precisa estar localizada dentro da região livre de interferência intersimbólica (do inglês, inter-symbol inteference) (ISI) (vide Fig. 2).

\section{A. Técnica \#1}

A técnica de sincronismo temporal discutida em [4] corresponde a uma adaptação daquela proposta em [7] ao contexto de metodologia de medição de canais PLC. Tal técnica baseia-se na correlação entre o CP e sua cópia em um símbolo OFDM, de forma que a localização do pico dessa correlação provê a estimativa de $n_{\text {sinc }}$. Diferentemente de utilizar um único símbolo OFDM para sincronização, como em [7], Oliveira et al. [4] faz uso de uma sequência de referência formada por sucessivos frames, compostos por dois símbolos HS-OFDM distintos em cada frame, $\mathbf{x}_{c p, 1} \in \mathbb{R}^{\left(2 N+L_{c p}\right) \times 1}$ e $\mathbf{x}_{c p, 2} \in$ $\mathbb{R}^{\left(2 N+L_{c p}\right) \times 1}$, i.e.,

$$
\begin{aligned}
x_{c p}[n]= & \sum_{j=-\infty}^{\infty} \sum_{i=0}^{1} \sum_{l=0}^{2 N+L_{c p}-1} x_{c p, i+1}[l] \times \\
& \delta\left[n-i\left(2 N+L_{c p}\right)-j\left(4 N+2 L_{c p}\right)\right],
\end{aligned}
$$

conforme ilustrado na Fig. 2(a). Sendo assim, considerando a sequência recebida pelo receptor $\{y[n]\}$, a correlação entre os sucessivos CPs e suas respectivas cópias é dada por

$$
p_{1}[n]=\sum_{l=0}^{L_{c p}-1} y[n+l] y[n+l+2 N]
$$




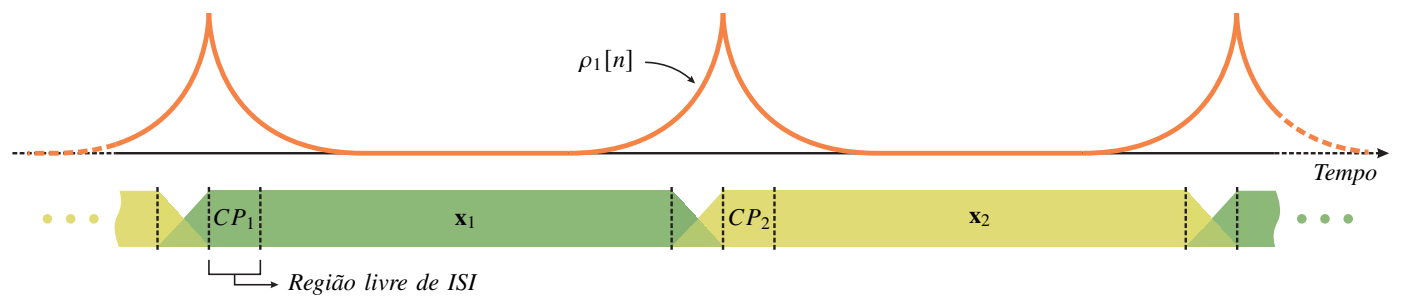

(a)

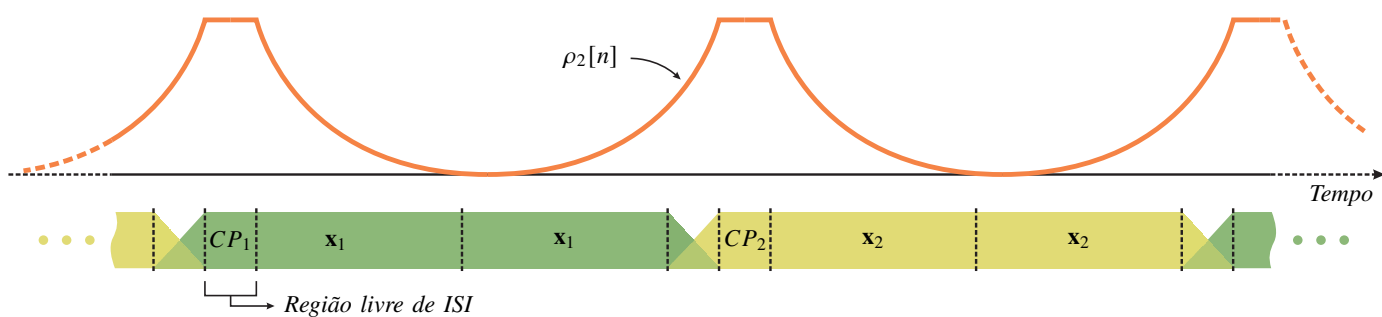

(b)

Fig. 2. Sinais de referência com suas respectivas funções de correlação normalizadas. (a) Técnica \#1. (b) Técnica \#2.

De acordo com [8], a normalização de (6) pode ser expressa por

$$
\rho_{1}[n]=\frac{p_{1}[n]}{r_{1}[n]},
$$

em que

$$
r_{1}[n]=\frac{1}{2} \sum_{m=0}^{1} \sum_{l=0}^{L_{c p}-1}|y[n+l+m 2 N]|^{2}
$$

representa a energia normalizada de (6). O comportamento de $\left\{\rho_{1}[n]\right\}$ ao longo da sequência de referência coletada pelo receptor $\{y[n]\}$ é retratado na Fig. 2(a).

As primeiras estimativas do início de cada símbolo HS-OFDM da sequência de referência são dadas por

$$
\hat{n}_{\text {sinc }, 1}^{\prime \prime}[i]=\arg \max _{n}\left\{\rho_{1, i}[n]\right\},
$$

de maneira que

$$
\rho_{1, i}[n]=\sum_{m=0}^{L_{\rho_{1}}-1} \rho_{1}[m] \delta\left[n+i\left(2 N+L_{c p}\right)-m\right],
$$

com $n=0,1, \ldots 2 N+L_{c p}-1$ e $L_{\rho_{1}}$ sendo o comprimento de $\left\{\rho_{1}[m]\right\}$.

No intuito de refinar $\left\{\hat{n}_{\operatorname{sinc}, 1}^{\prime \prime}[i]\right\}$, Oliveira et al. [4] propôs uma métrica, a qual pode ser representada pela expressão

$$
\begin{aligned}
m_{i}[r]=\frac{1}{K_{d}} \sum_{p=1}^{K_{d}}\left(y\left[\hat{n}_{\text {sinc }, 1}^{\prime \prime}[i]-\frac{R}{2}+r-p\right]-\right. \\
\left.y\left[\hat{n}_{\text {sinc }, 1}^{\prime \prime}[i]-\frac{R}{2}+2 N+r-p\right]\right)^{2},
\end{aligned}
$$

em que $r=0,1, \ldots, R-1, R \in \mathbb{Z}_{+}$e $m_{i}[r]$ retorna o erro quadrático médio entre as $K_{d}$ amostras que antece$\operatorname{dem} \hat{n}_{\text {sinc }, 1}^{\prime \prime}[i]$ e $\hat{n}_{\text {sinc }, 1}^{\prime \prime}[i]+2 N$ nos respectivos intervalos $\left[\hat{n}_{\text {sinc }, 1}^{\prime \prime}[i]-R / 2, \hat{n}_{\text {sinc }, 1}^{\prime \prime}[i]+R / 2\right]$ e $\left[\hat{n}_{\text {sinc }, 1}^{\prime \prime}[i]+2 N-\right.$ $\left.R / 2, \hat{n}_{\text {sinc }, 1}^{\prime \prime}[i]+2 N+R / 2\right]$. Dessa forma, as estimativas refinadas são dadas por

$$
\hat{n}_{\text {sinc }, 1}^{\prime}[i]=\hat{n}_{\text {sinc }, 1}^{\prime \prime}[i]-\arg \min _{r}\left\{m_{i}[r]\right\},
$$

de forma que, $\arg \min _{r}\left\{m_{i}[r]\right\}$ define o menor índice do vetor $\mathbf{m}_{i} \in \mathbb{R}_{+}^{R \times 1}$. Posto isto, a estimativa do ponto inicial da sequência de referência é obtida por meio de

$$
\hat{n}_{s i n c, 1}=\frac{1}{J} \sum_{i=0}^{J-1} \hat{n}_{\operatorname{sinc,1}}^{\prime}[i]+L_{c p},
$$

em que $J$ é o comprimento de $\left\{\hat{n}_{\text {sinc, },}^{\prime}[i]\right\}$. É importante destacar que, conforme discutido em [4], a obtenção de $\hat{n}_{\text {sinc }, 1}$ através da média dos pontos iniciais de cada símbolo HS-OFDM tem como consequência a mitigação da influência do ruído aditivo gaussiano branco (do inglês, additive white Gaussian noise) (AWGN) durante o processo de sincronização de símbolo.

\section{B. Técnica \#2}

Em [6], os autores propuseram uma técnica de sincronização de símbolo baseada no esquema OFDM. Basicamente, através de um único símbolo OFDM com metades iguais alocado em um preâmbulo, o ponto inicial do sinal transmitido é estimado no receptor através da correlação entre essas duas metades. Observe que para obter um símbolo OFDM com duas metades iguais, os autores transmitem informação apenas nas subportadoras pares, enquanto que nas subportadoras ímpares transmitem zero.

Entretanto, no intuito de aplicar a ideia proposta em [6] na metodologia de medição discutida em [4], o sinal de referência não é gerado da mesma forma que o preâmbulo de [6]. Em vez de se utilizar apenas um único símbolo OFDM com metades iguais, considera-se dois símbolos HS-OFDM sucessivos iguais para que, além da sincronização, eles possam ser utilizados para a estimação do canal PLC. Além disso, no intuito de mitigar o ruído AWGN, considera-se o sinal de referência composto por frames consecutivos contendo

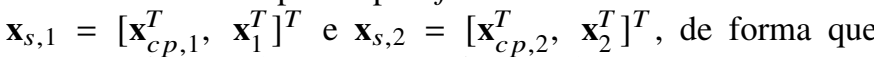
$\mathbf{x}_{s, 1} \in \mathbb{R}^{4 N+L_{c p} \times 1}$ e $\mathbf{x}_{s, 2} \in \mathbb{R}^{4 N+L_{c p} \times 1}$, como ilustrado na Fig. 2(b). Assim, a sequência de referência pode ser expressa 
como

$$
\begin{aligned}
x_{c p}[n]= & \sum_{j=-\infty}^{\infty} \sum_{i=0}^{1} \sum_{l=0}^{4 N+L_{c p}-1} x_{s, i+1}[l] \times \\
& \delta\left[n-i\left(4 N+L_{c p}\right)-j\left(8 N+2 L_{c p}\right)\right] .
\end{aligned}
$$

Dessa forma, a partir da sequência $\{y[n]\}$ coletada pelo receptor, calcula-se a correlação entre duas janelas consecutivas de tamanho $2 \mathrm{~N}$ dada por

$$
p_{2}[n]=\sum_{l=0}^{2 N-1} y[n+l] y[n+l+2 N] .
$$

Observa-se que a energia referente a segunda janela pode ser expressa por

$$
r_{2}[n]=\sum_{l=0}^{2 N-1}|y[n+l+2 N]|^{2},
$$

em que $|\cdot|$ é o operador valor absoluto, de modo que, de acordo com [6], a normalização de (15) pode ser escrita como

$$
\rho_{2}[n]=\frac{p_{2}[n]}{r_{2}[n]} .
$$

Nota-se que as janelas consideradas para calcular (15) deslizam por toda a sequência recebida no receptor, $\{y[n]\}$, de modo que, quando as mesmas passam pela região livre de ISI, $p_{2}[n]$ assume a forma de um platô (vide Fig. 2(b)), com comprimento igual a $L_{c p}$ menos o comprimento da CIR, $L_{h}$. Sendo assim, para estimar $n_{\text {sinc }}$ no contexto da metodologia de medição, faz-se necessário a utilização do limiar $\rho_{t h}=$ $0.9 \max \left\{\rho_{i}[n]\right\}$. Dessa forma, o inicio dos símbolos $\mathbf{x}_{s, 1} \mathrm{e}$ $\mathbf{x}_{s, 2}$, assim como suas réplicas, pode ser estimado por

$$
\hat{n}_{\text {sinc }, 2}^{\prime}[i]=\frac{1}{2}(\max \{m\}+\min \{m\})
$$

em que $m=\left\{n \in \mathbb{N} \mid \rho_{2, i}[n]>\rho_{t h}\right\}$,

$$
\rho_{2, i}[n]=\sum_{m=0}^{L_{\rho_{1}}-1} \rho_{2}[m] \delta\left[n+i\left(4 N+L_{c p}\right)-m\right],
$$

com $n=0,1, \ldots, 4 N+L_{c p}$ e $L_{\rho_{1}}$ sendo o comprimento de $\rho_{2}[n]$. Por fim, a estimativa de $n_{\text {sinc }}$ é dada por

$$
\hat{n}_{\text {sinc }, 2}=\frac{1}{J} \sum_{i=0}^{J-1} \hat{n}_{\text {sinc }, 2}^{\prime}[i],
$$

em que $J$ denota o comprimento de $\left\{\hat{n}_{\text {sinc }, 2}^{\prime}[i]\right\}$.

\section{RESUltados NumÉRicos}

Essa seção apresenta análises numéricas comparativas entre as técnicas de sincronização de símbolo \#1 e \#2. As técnicas são comparadas através da média e variância dos pontos de sincronismo estimados $\left\{\hat{n}_{\text {sinc, }}^{\prime}[i]\right\}, \operatorname{com} q \in\{1,2\}$ representando as técnicas \#1 e \#2, respectivamente. Observa-se que para a técnica \#1, adota-se $K_{d}=8$ e $R=128$, conforme apresentado em [4], e $K_{d}=256$ e $R=1024$ com base em testes realizados em ambiente de simulação. Além disso, para a simulação numérica, considera-se uma estimativa de canal PLC banda larga obtida através de campanha de medição

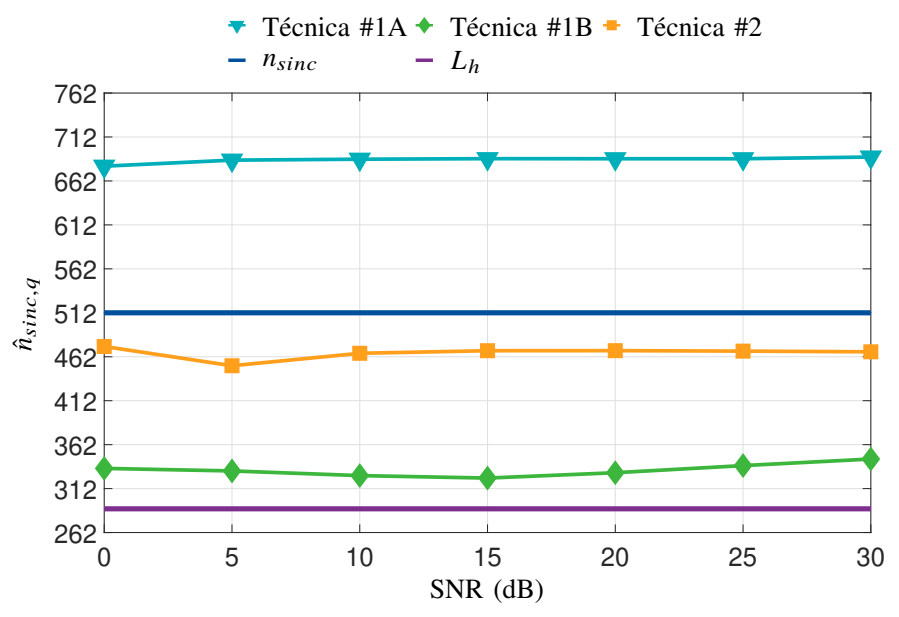

Fig. 3. Estimativas dos pontos de sincronismo $\hat{n}_{\text {sinc,q }}$ obtidas através das técnicas \#1 e \#2.

realizada em redes de energia elétrica internas a residências [9]. Note que essa estimativa possui $L_{h}=289$ e cobre a faixa de frequências entre 1.7 MHz e $100 \mathrm{MHz}$. Além disso, o ruído é modelado como AWGN. Em relação à geração do sinal de referência, adota-se símbolos HS-OFDM gerados a partir de uma modulação por deslocamento de fase (do inglês, binary phase-shift keying) (BPSK), com $N=2048$ e $L_{c p}=512$.

Na Fig. 3, as estimações dos pontos de sincronismo $\hat{n}_{s i n c, q}$ obtidas através das técnicas \#1 e \#2 são apresentadas em termos de SNR. A respeito da técnica \#1, nota-se que o rótulo técnica \#1A representa a escolha $K_{d}=8$ e $R=128$, enquanto que o rótulo técnica \#1B denota a escolha $R=256 \mathrm{e}$ $K_{d}=1024$. Observando a figura, pode-se notar que a técnica \#1A é bastante afetada pelo espalhamento do atraso do canal PLC, de forma que valores de $\hat{n}_{\text {sinc, } 1 \mathrm{~A}}$ em torno de 687 são observados. Em contrapartida, a técnica \#1B e a técnica \#2 mantiveram-se na região livre de ISI, de maneira que $\hat{n}_{s i n c, 1 \mathrm{~B}}$ próximo a 350 e $\hat{n}_{s i n c, 2}$ em torno de 462 são observados. De forma geral, pode-se constatar que a técnica \#1 é fortemente afetada pela escolha dos parâmetros $K_{d}$ e $R$. Enquanto que as estimativas $\hat{n}_{\text {sinc, } 1 \mathrm{~B}}$ da técnica \#1B estavam dentro da região livre de ISI, as da técnica \#1A estavam fora dessa zona.

Na Fig. 4, são mostradas as variâncias das estimações dos pontos de sincronismo $\left\{\hat{n}_{\text {sinc, },}^{\prime}[i]\right\}$ em termos de SNR para as técnicas \#1 e \#2. Nota-se que a curva relacionada à técnica \#2 apresenta uma dispersão bem menor se comparada com as curvas das técnica \#1A e \#1B para valores de SNR maiores que $10 \mathrm{~dB}$. De fato, é observada uma variância $\approx 10^{2}$ quando a SNR é igual $10 \mathrm{~dB}$ e de $\approx 10$ para uma SNR de $30 \mathrm{~dB}$. Já em relação à técnica \#1, observa-se variâncias próximas a $10^{4}$ e $10^{3}$ quando a SNR é igual a $10 \mathrm{~dB}$ e em torno de $10^{3}$ e $10^{2}$ quando a SNR é igual a $30 \mathrm{~dB}$ para as técnicas \#1A e \#1B, respectivamente.

\section{CONSIDERAÇÕES FINAIS}

Neste trabalho foi proposta uma adaptação da técnica de Schmidl \& Cox [6] para aplicação na etapa de sincronização de símbolo da metodologia de estimação de canais PLC apresentada em [4], rotulada nesse trabalho como técnica 


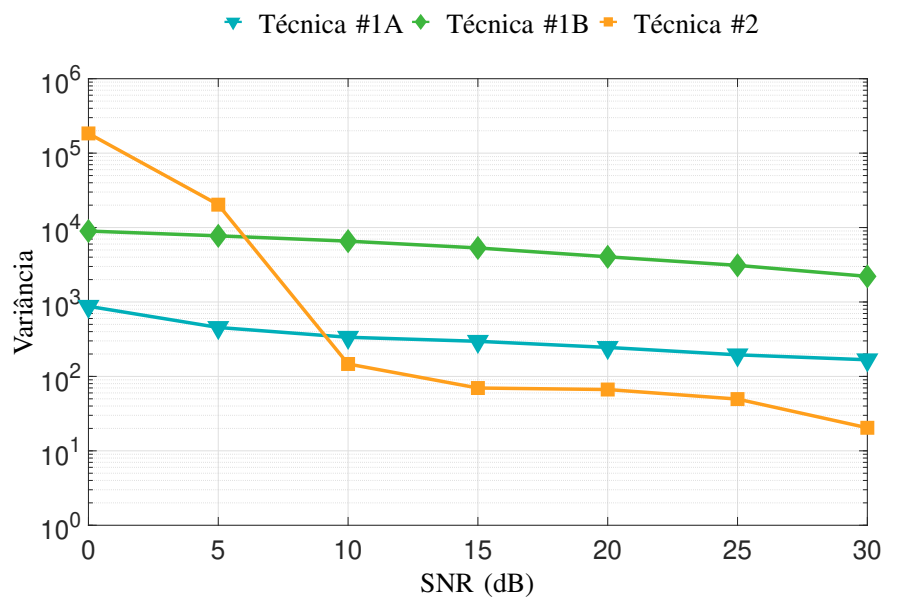

Fig. 4. Variância das estimativas dos pontos de sincronismo $\left\{\hat{n}_{\text {sinc,q }}^{\prime}[i]\right\}$ obtidos através das técnicas \#1 e \#2.

\#2. Sendo assim, resultados numéricos comparativos entre a técnica \#2 e a técnica de sincronismo temporal discutida em [4], rotulada como técnica \#1, foram apresentados.

A partir dos resultados numéricos, observou-se que o desempenho da técnica \#1 é fortemente afetado pela escolha dos parâmetros $K_{d}$ e $R$, sendo essa escolha determinante para o funcionamento adequado da técnica. Já a técnica \#2, além de não necessitar do ajuste de qualquer parâmetro empírico, apresentou resultados bastante promissores, mostrando-se mais efetiva que a técnica \#1 no cenário simulado. De forma geral, os resultados obtidos apontam que a técnica \#2 pode oferecer mais benefícios que a técnica \#1 quando aplicada na etapa de sincronização de símbolo da metodologia de estimação de canais PLC.

\section{REFERÊNCIAS}

[1] H. Gassara, F. Rouissi, and A. Ghazel, "Statistical characterization of the indoor low-voltage narrowband power line communication channel," IEEE Transactions on Electromagnetic Compatibility, vol. 56, no. 1, pp. 123-131, Feb. 2014.

[2] Y. Xiaoxian, Z. Tao, Z. Baohui, H. Zonghong, C. Jian, and G. Zhiqiang, "Channel model and measurement methods for 10-kv medium-voltage power lines," IEEE transactions on power delivery, vol. 22, no. 1, pp. 129-134, 2006.

[3] J. D. Parsons, D. A. Demery, and A. M. D. Turkmani, "Sounding techniques for wideband mobile radio channels: a review," IEEE Proceedings in Communications, Speech and Vision, vol. 138, no. 5, pp. 437-446, Oct. 1991.

[4] T. R. Oliveira, C. A. G. Marques, W. A. Finamore, S. L. Netto, and M. V. Ribeiro, "A methodology for estimating frequency responses of electric power grids," Journal of Control, Automation and Electrical Systems, vol. 25, no. 6, pp. 720-731, Dec. 2014.

[5] C. Tzi-Dar Cheueh and Pei-Yun Tsai, OFDM Baseband Receiver Design for Wireless Communications. John Wiley \& Sons, 2007.

[6] T. M. Schmidl and D. C. Cox, "Robust frequency and timing synchronization for ofdm," IEEE Transactions on Communications, vol. 45, no. 12, pp. 1613-1621, Dec. 1997.

[7] J. . van de Beek, P. O. Borjesson, M. . Boucheret, D. Landstrom, J. M Arenas, P. Odling, C. Ostberg, M. Wahlqvist, and S. K. Wilson, "A time and frequency synchronization scheme for multiuser ofdm," IEEE Journal on Selected Areas in Communications, vol. 17, no. 11, pp. 1900-1914, Nov. 1999.

[8] J. Godoy, F. J. Cañete, J. A. Cortés, and L. Díez, "A study on symbol timing recovery schemes for broadband in-home PLC," in 2012 IEEE International Symposium on Power Line Communications and Its Applications, 2012, pp. 188-193.
[9] T. R. Oliveira, A. A. M. Picorone, S. L. Netto, and M. V. Ribeiro, "Characterization of Brazilian in-home power line channels for data communication," Electric Power Systems Research, vol. 150, pp. 188197, 2017. 